FLYOVER COUNTRY 
Princeton Series of Contemporary Poets

Susan Stewart, series editor

For other titles in the Princeton Series of Contemporary Poets see page 115 


\section{FLYOVER COUNTRY}

Poems

Austin Smith

PRINCETON UNIVERSITY PRESS

Princeton and Oxford 
Copyright (C) 2018 by Princeton University Press

Published by Princeton University Press

41 William Street, Princeton, New Jersey 08540

6 Oxford Street, Woodstock, Oxfordshire OX20 1TR

press.princeton.edu

All Rights Reserved

Library of Congress Control Number: 2018930998

ISBN: 978-0-691-18156-1

ISBN (pbk.) 978-0-691-18157-8

British Library Cataloging-in-Publication Data is available

Acquisitions Editor: Anne Savarese

Editorial Assistant: Thalia Leaf

Production Editorial: Ellen Foos

Text and cover design: Pamela Schnitter

Cover image: Aerial view above pivot irrigation circles,

Edward County, Kansas. Aerial Archives / Alamy Stock Photo

Production: Jacqueline Poirier

Copyeditor: Jodi Beder

This book has been composed in Adobe Garamond Pro and ScalaSans OT

Printed on acid-free paper. $\infty$

Printed in the United States of America

1098765543321 
For Holly Mulder-Wollan 
